\title{
The Impact of Strategy Training on Vocabulary Comprehension
}

\author{
Yougen Lou', Pei Xu' \\ ${ }^{1}$ School of Foreign Studies, Yangtze University, Jingzhou, China \\ ${ }^{2}$ Paotong Elementary School, Wuhan, China \\ Email: louyougen@163.com
}

Received 14 November 2015; accepted 14 March 2016; published 17 March 2016

Copyright (C) 2016 by authors and Scientific Research Publishing Inc.

This work is licensed under the Creative Commons Attribution International License (CC BY).

http://creativecommons.org/licenses/by/4.0/

(c) (i) Open Access

\section{Abstract}

This article focuses on a study of the effect of strategy training on vocabulary comprehension. 112 sophomore English-majored students at Yangtze University were chosen as participants in a strategies-based instructional treatment or comparison group students receiving the regular vocabulary course. Data were gained through the performance of a set of two vocabulary tasks on a prepost basis by both groups and were analyzed by the tool SPSS17.0. The subsample of 24 students also provided verbal report data to show their cognitive insights into strategy use and the instruction itself. The findings showed that 1) Chinese English-majors used a variety of English vocabulary learning strategies in vocabulary learning, including metacognitive, cognitive and social/affective strategies. 2) The increased use of vocabulary strategies contributed positively to vocabulary and article comprehension.

\section{Keywords}

Impact, Strategy Training, Vocabulary

\section{Introduction}

Vocabulary learning is the greatest stumbling block for most language learners. As an indispensable part of language system, vocabulary learning is of great importance to language acquisition. Vocabulary is a very important component of a language.

This article focuses on the impact of strategy training on vocabulary comprehension. It briefly lists literature related to this issue, and then considers a study conducted at Yangtze University. 112 sophomore English-majored learners at Yangtze University were chosen as participants in a strategies-based instructional treatment or comparison group students receiving the regular vocabulary course in the study. Both groups filled out a pre- 
treatment questionnaire completed a set of two vocabulary tasks on a pre-post basis. The experimental group also reported the frequency of their use of specific strategies. 24 out of the total 112 students were further to give verbal report data which displayed their insights into the strategy use and their reactions towards the instruction.

This article was to examine the impact that formal strategy training might have on learners in university-level foreign language classrooms, with a particular focus on vocabulary, because this field had received limited attention in research literature on strategy training. This study attempted to answer the following questions:

1) What types of strategies do English-majored students tend to employ in learning vocabulary?

2) How does strategy training affect students’ vocabulary proficiency?

3) How do those strategies help students while they perform the vocabulary tasks?

\section{Literature Review}

Research on learning strategies can be divided into three stages. The first stage concerns with identifying and listing all the strategies that foreign language learners used in their learning. In the second stage, researchers focus on classifying and giving definitions to strategies. The third stage deals with the effectiveness of learning strategies with their results most fascinating because there are many classifications and definitions.

The literature on learning strategies in foreign language learning emerged from a concern for identifying the characteristic of effective learners. Research focusing on the "good language learners" had identified strategies reported by students or observed in language learning situations that seemingly contributed to learning. These efforts demonstrated that students do apply learning strategies while learning a foreign language and these strategies can be described and classified. Therefore, many researchers [1]-[3] studied the identification and classification of learning strategies.

Influenced strongly by the findings of cognitive psychology, O’Malley and Chamot [2] considered learning strategies as cognitive skills. Learning strategies have been divided into three categories according to the level or type of processing involved [2]. They are meta-cognitive strategies, cognitive strategies, and social/affective strategies. Meta-cognitive strategies are higher order executive skills that may entail planning for, monitoring, or evaluating the success of a learning activity. Cognitive strategies operate directly on incoming information, manipulating it in ways that enhance learning. Social/affective strategies represent a broad grouping that involves either interaction with another person or ideational control over affect.

The following is the greatest differentiation and heaviest use in subcategories of planning strategies (Table 1).

\section{Methods}

\subsection{Participants}

From February, 2014 to July, 2014, 112 second-year English-majored students at Yangtze University participated in this study. They were from four intact classes. Two classes of 56 comprised the comparison group and received regular vocabulary instruction. Other two classes of 56 students served as the experimental group and received strategies-based vocabulary instruction. Twenty four students out of the total 112 were selected on a volunteer basis to provide additional data in the form of verbal report protocols about their strategy use. These students represented three different levels of vocabulary ability in their respective classes (8 high-level, 8 mid-level and another 8 low-level). Among the 112 subjects, 59 were females and 53 males, average age 19 with Chinese as the main language. Their majors were chemistry, agriculture, plant protection, finance, biological technology. All the 112 second-year English-majored students were taught by one teacher. The students in the comparison group were taught by regular vocabulary instruction that 2 hours per week, total 30 hours 15 weeks, the students followed the teacher to learn Chinese and English meanings of new words on the words list in the textbooks without other strategies on vocabulary comprehension trained by the teacher. While the students in the experimental group received strategies-based vocabulary instruction that 2 hours per week, total 30 hours 15 weeks, the teacher explained meta-cognitive strategies, cognitive strategies and social/affective strategies to students in the experimental group and trained students in the experimental group how to use these strategies to improve vocabulary comprehension in details. For example, students in the experimental group were trained to apply the "contextualization" strategy and the "word formation" strategy to analyze the context and methods of word formation to guess the meanings of new words in the paper. 
Table 1. Learning strategies.

\begin{tabular}{|c|c|}
\hline Strategies items & Description \\
\hline \multicolumn{2}{|l|}{ Metacognittive Strategies } \\
\hline Advanced organizers & $\begin{array}{l}\text { Making a general but comprehensive preview of the concept or principle in an anticipated learning } \\
\text { activity. }\end{array}$ \\
\hline Direct attention & Deciding in advance to attend in general to a learning task and to ignore irrelevant distracters. \\
\hline Selective attention & $\begin{array}{l}\text { Deciding in advance to attend specific aspects of language input or situational details that will cue the } \\
\text { retention of language input. }\end{array}$ \\
\hline Advance preparation & Planning for and rehearsing linguistic component necessary to carry out an upcoming language task \\
\hline Self-monitoring & $\begin{array}{l}\text { Correcting one's speech for accuracy in pronunciation, grammar, vocabulary, or for appropriateness } \\
\text { related to the setting or to the people who are present. }\end{array}$ \\
\hline Self-evaluation & $\begin{array}{l}\text { Checking the outcomes of his language learning against an internal measure of completeness and accu- } \\
\text { racy. }\end{array}$ \\
\hline \multicolumn{2}{|l|}{ Cognitive Strategies } \\
\hline Repetition & Imitating a language model, including overt practice and silent rehearsal. \\
\hline Recoursing & Defining or expanding a definition of a word or concept through use of target reference material. \\
\hline Direct physical response & Relating new information to physical actions as directive. \\
\hline Translation & Using the first language as a base for understanding and/or producing the second language. \\
\hline Grouping & Reordering or reclassifying and perhaps labeling the material to be learned based on common attributes. \\
\hline Note-taking & $\begin{array}{l}\text { Writing down the main idea, important points, outline, or summary of information presented orally or } \\
\text { in writing. }\end{array}$ \\
\hline Deduction & Consciously applying rules to produce or understand the second language. \\
\hline Imagery & $\begin{array}{l}\text { Relating new information to visual concepts in memory via familiar easily retrievable visualization, } \\
\text { phrases, or locations. }\end{array}$ \\
\hline Contextualization & Placing a word or phrase in a meaningful language sequence. \\
\hline Elaboration & Relating new information to other concepts in memory. \\
\hline Transfer & $\begin{array}{l}\text { Using previously acquired linguistic and/or conceptual knowledge to facilitate a new language learning } \\
\text { task. }\end{array}$ \\
\hline Inferencing & $\begin{array}{l}\text { Using available information to guess meanings of new items, predict outcomes, or fill in missing } \\
\text { information. }\end{array}$ \\
\hline \multicolumn{2}{|l|}{ Social/affective } \\
\hline Cooperation activity. & Working with one or more peers to obtain feedback, pool information, or model a language \\
\hline Question for classification & Asking a teacher or other native speaker for repetition, paraphrasing, explanation, and/or examples. \\
\hline
\end{tabular}

(O’Malley and Chamot's classification of learning strategies, 1990).

\subsection{Instrumentation}

The instruments used in this study were a questionnaire, vocabulary task battery and verbal report protocols.

All subjects were administered a pre-treatment questionnaire to measure their knowledge of vocabulary strategies, their use of these strategies and their perception of the value of the strategies. Then the teacher and the experimental students together worked out a list of strategies useful for the students' vocabulary task performance based on O'Malley and Chamot's [2] model. The list mainly included (chapter 2 literature review).

\subsubsection{Vocabulary Task Battery}

A Vocabulary Task Battery was designed and made up of a series of two vocabulary tasks. All subjects from the comparison and experimental groups were asked to complete the similar two tasks on a pre-test and post-test basis to determine whether there were gains in vocabulary ability for the experimental students over the 16-week 
training. The vocabulary materials used in this study were taken from An Introduction to Modern English Lexicology (published by Beijing Normal University Press), so they were almost at the same level of difficulty. And two different types of vocabulary tasks (a news report and a vocabulary test) were expected to elicit a range of vocabulary strategies, such as selective attention, elaboration, keyword, etc.

\subsubsection{Verbal Report Protocols}

The post-test data collection also included an extra feature for the 24 students from both the comparison and experimental groups (representing high, medium, and low proficiency in vocabulary, as determined through teacher ratings). These subjects were first asked to describe what was going on in their minds before, during and after the vocabulary learning process, and what strategies they used, and finally why they used certain specific strategies.

\subsection{Data Collection Procedures}

The different vocabulary tasks were rated according to a set of five points, multi-trait scales. Data obtained from the pre-test and post-test vocabulary tasks were used to determine students' improvement in vocabulary proficiency. Post-test means were compared with the pre-test means. Pre-test and post-test gains on the vocabulary tasks were inputted the SPSS17.0 to be analyzed in relation to pretest and post-test gains in the reported use of strategies for the given tasks which is represented by students' self-ratings of the frequency of use of different strategies. In other words, the effect of increase frequency of use of a given strategy was calculated by correlating the gains in vocabulary performance in general.

The verbal report data were analyzed separately from the vocabulary task data, and aimed at students' insights into strategy use and feedback of the instruction. This verbal report served as a qualitative means for making sure whether the training was reliable and valid, and the results were reported in Table 3.

\section{Results}

\subsection{Results of English Vocabulary Learning Strategies Use}

The first investigated question is what the whole pattern of EVLS employed by the Chinese English-majors investigated. The questionnaire is a Five-point scale, so a mean score which is above 3 indicates high frequency in the use of corresponding strategy while a mean score is below 3, which represents low Frequency in the use of the strategy .The higher the mean score is, the more often the strategy is used. In order to determine how they avail themselves to each vocabulary learning strategy, average mean score and standard deviation and strategies use at three levels are calculated (see Table 2).

Firstly, strategy use was at meta-cognitive Level. It was obvious that selective attention( Mean $=3.48, \mathrm{~S}=$ 1.04) was the highest rank among the four categories of meta-cognitive strategies, which indicates that the investigated subjects are positive with regard to identifying important to-be-learned words and thus sparking their consciousness to acquire these words. Learner autonomy (Mean $=2.98, \mathrm{~S}=1.05)$, Planning (Mean $=3.10, \mathrm{~S}=$ 1.18), which means English-majors adopt often to plan their English vocabulary learning. Self-evaluation (Mean = 3.08, $\mathrm{S}=1.06$ ), which means English-majors can evaluate their English vocabulary for them to have time make changes about vocabulary learning. Reasons to account for the English-majors meta-cognitive strategies were that English-majors are totally adults who know how to put their attention, learn English vocabulary autonomy without pushing them to learn, and make a plan to learn vocabulary then evaluate their learning procedure by themselves.

Secondly, strategy use was at cognitive strategies level. From Table 2, we can see that the rank order of English vocabulary learning strategies at cognitive level was translation, dictionary looking-up, repetition, word formation, contextualization, imagery, grouping, keyword strategies. It was evident that translation (Mean = $3.70, \mathrm{~S}=1.15$ ) ranks the highest among all the English vocabulary learning strategies at cognitive stage, in other words, which means that English-majors use translation as the most frequently English vocabulary learning strategy. Next followed by dictionary looking-up (Mean $=3.41, \mathrm{~S}=1.05)$ appears as the second frequently used cognitive strategy, which suggest that English-majors often learn the new words with the help of a dictionary. Repetition (Mean $=3.18, \mathrm{~S}=1.27$ ) was used by the more investigated subjects and it is evident that repetition demonstrates that more of the investigated subjects still have the conventional belief that vocabulary could be 
Table 2. Mean and standard deviation of strategies use at three levels.

\begin{tabular}{cccc}
\hline Dimensions & Categories & M & S. \\
& Selective attention & 3.48 & 1.04 \\
Learner autonomy & Planning & 2.98 & 1.05 \\
Strategies & Self-evaluation & 3.10 & 1.18 \\
& Word formation & 3.08 & 1.06 \\
Dictionary Looking-up & Keyword & 3.10 & 1.03 \\
Cognitive Strategies & Grouping & 3.41 & 1.05 \\
& Imagery & 2.19 & 1.06 \\
& Repetition & 3.07 & 1.05 \\
Translation & 2.81 & 1.04 \\
Cocial/ & Contextualization & 3.18 & 1.27 \\
Group work & 3.70 & 1.15 \\
& Affective control & 3.05 & 1.01 \\
\hline
\end{tabular}

remembered via repetition that was related to rote learning. This belief has deep roots in traditional Chinese ideas about learning. This conventional way of teaching and learning still has a powerful influence in China nowadays. So it was natural to find the root in modern English teaching and learning in university. At the same time, it should be noted that some other mnemonic strategies including: grouping (Mean $=3.07, \mathrm{~S}=1.05$ ) means that the investigated subjects learn vocabulary by group vocabulary into different classification; contextualization (Mean $=3.05, \mathrm{~S}=1.01$ ) suggest that students can guess the meaning of the new words with the help of the context; word formation (Mean $=3.10 \mathrm{~S}=1.03$ ) means the investigated subjects use it to help them learn English vocabulary; imagery (Mean $=2.81, \mathrm{~S}=1.04$ ) means students seldom use their imagery to help them learn the new words; keyword (Mean $=2.19, \mathrm{~S}=1.06$ ) suggests that students seldom use it to learn vocabulary. The higher value of dictionary looking-up indicates that the investigated subjects are becoming the mature dictionary users, which might be introduced by the relatively poor English foreign language learning environment in China. The investigated subjects comparably make more use of contextualization strategies when learning new words. Language proficiency may play an even greater role in determining a vocabulary strategies' effectiveness. When learners' language proficiency reaches the threshold, some English learning strategies will be effective, such as guessing the new words' meaning from the context, using word formation to remember new words.

Finally, Social/Affective strategies are concerned, group work (Mean $=3.16, \mathrm{~S}=0.94)$ ranks higher than affective control (Mean $=3.06, \mathrm{~S}=0.99$ ). English majors could be as a group to discuss a topic.

\subsection{The Effect of Strategy Training on Vocabulary Proficiency}

On the effect of strategy training on vocabulary proficiency, the results of pre-test and post-test means showed that the performance of the comparison group was less than that of the experimental group on all the two tasks (see Table 3). And the correlation data suggested a significant relationship between the increased use of strategies and task performance (see Table 4).

\subsection{Students' Use of the Instructed Strategies}

Verbal report data collected from the students was intended to measure the effect of strategy training from cognitive perspective. The verbal report protocols yielded two types of data-insights about students' strategy use as well as their reactions towards such training program, as presented below. 
Table 3. Overall vocabulary performance by task Pre-test and Post-test means.

\begin{tabular}{|c|c|c|c|c|}
\hline & \multicolumn{2}{|c|}{ Task 1 A news report } & \multicolumn{2}{|c|}{ Task 2 A vocabulary test } \\
\hline & Pre-test Mean & Post-test Mean & Pre-test Mean & Post-test Mean \\
\hline $\begin{array}{l}\text { Comparison Group } \\
\qquad(\mathrm{N}=56)\end{array}$ & 3.91 & 3.96 & 3.67 & 3.74 \\
\hline $\begin{array}{l}\text { Experimental Group } \\
\qquad(\mathrm{N}=56)\end{array}$ & 3.98 & 4.47 & 3.54 & 4.01 \\
\hline
\end{tabular}

Table 4. Gain in task performance in general correlated with gain in reported frequency of strategy use by experimental students.

\begin{tabular}{cccc}
\hline Vocabulary Strategies & Pre-test & Post-test & performance \\
\hline Selective attention & 3.35 & 4.36 & 0.50 \\
Learner autonomy & 3.01 & 4.21 & 0.48 \\
Self-evaluation & 2.77 & 3.99 & 0.41 \\
Word formation & 2.78 & 4.02 & 0.45 \\
Dictionary Looking-up & 3.12 & 4.59 & 0.59 \\
Repetition & 3.32 & 4.56 & 0.56 \\
Translation & 3.45 & 4.56 & 0.58 \\
Elaboration & 3.54 & 4.49 & 0.56 \\
\hline
\end{tabular}

\subsubsection{Comparison Group}

Pre-test

"I thought if I could understand every word I could understand the sentence, the paragraph and the whole article.”

"It's easier to understand something you're familiar with."

Post-test

"When I met a new word in reading an article, I become very nervous."

"I have difficulty understanding all these strategies. I have never known them before."

\subsubsection{Experimental Group}

\section{Pre-test}

"Never thought of using specific strategies to help understand more."

"Tried to understand every word as well as the main idea."

Post-test

"How awful my vocabulary comprehension ability is. I can apply the "word formation" strategy, the "contextualization" to improve my vocabulary comprehension for some new words in an exam; I may apply the "dictionary looking-up" strategy to improve my vocabulary comprehension for some new words in reading papers, books, newspapers etc., I will turn to a dictionary to know the meanings of new words I come cross.

"I want more training so as to make full use of the strategies. Before strategy training on vocabulary comprehension, I just know to look up a dictionary for the meanings of new words, but in an exam, I cannot check the meanings of the new words I meet in a dictionary. Through strategy training on vocabulary comprehension, I may apply meta-cognitive strategies, cognitive strategies, and social/affective strategies. These strategies help me improve my vocabulary comprehension, but I don't know when and how to make full use of them. So more strategy training will be better for me.”

\section{Discussion}

Table 2 showed us that students used different strategies and some students adopted more vocabulary strategies to help them understand the article. But one big problem was found that the students were poor at using me- 
ta-cognitive strategies. They were less to plan their learning in vocabulary and learned vocabulary by their autonomy. The results in Table 2 are generally equal to those of Gu and Johson's study except the following parts: one was the present study shows that the English-majors investigated are more positive in using the meta-cognitive strategy—selective attention than learner autonomy at meta-cognitive strategy, which is in contrast with $\mathrm{Gu}$ and Johson's study; the other was that the present study indicates that rote learning strategies (repetition) plays an important role in students' vocabulary learning process while Gu and Johson's [4] study suggests that students don't value rote learning as highly as other strategies. The difference may result from the difference of the investigated subjects.

The question of whether strategy training affects vocabulary performance, the results seemed to be positive: the comparison group was less than the experimental group on all the two tasks. It was understandable that dictionary looking-up was the highest correlation coefficient among all the strategies in Table 4, because it was the easiest one to get the meaning of a new word. It seemed that other strategies would enhance vocabulary comprehension ability. For the comparison, the pre-test and post-test mean difference was too small to be counted. The verbal report showed that strategy training did help students with their vocabulary and article comprehension. From the comments of the post-test experimental group, as compared with that of the comparison group, we found that students, having learned, seemed to be helpful for their language learning.

It seemed clear that the students in the comparison group could adopt some strategies without training, however, seeming unconsciously and very basic. But it does not mean that the strategies instruction is unnecessary, for some students from the comparison group shared the experience that they never knew and were not familiar with the strategies in the list and hoped to learn strategies to enhance their vocabulary and article comprehension ability.

This study gave suggestions that it would be beneficial to engage learners in learning of vocabulary strategies and adopted those strategies in class. If the teachers introduce systematically and reinforce strategies, the students might learn more effectively.

The findings showed that 1) Chinese English-majors used a variety of English vocabulary learning strategies in vocabulary learning, including meta-cognitive, cognitive and social/affective strategies. 2) The increased use of vocabulary strategies contributed positively to vocabulary and article comprehension.

\section{Limitations and Suggestions}

Though the present study has provided a comparatively detailed description of strategies training on vocabulary comprehension applied among 112 second-year English-majored students, there are still some limitations of the study. Having the limitations in mind, suggestions for further research, therefore, can be put forward at the same time in order to achieve a lot thorough understanding of strategies training on vocabulary.

Firstly, being time limitation (only five months) and other practical restrictions such as the subjects in the study consisted of only 112 second-year English-majored students in one university need to be broadened in further research.

Secondly, the instruments used to investigate English-majored students of strategies training on vocabulary comprehension involve structured questionnaire and tests. The study would be much better, if it were combined with other instruments such as observation. More instruments should be used in investigation in further research.

Finally, strategies training on vocabulary comprehension applied in second-year English-majored students can improve English-majored students' English vocabulary comprehension, but strategies training on vocabulary comprehension also works for other subjects such as graduate students, we need further research.

Despite of the restraints of the study, it is hoped that it can offer some guidelines for further research on strategies training on vocabulary comprehension applied in English teaching.

\section{References}

[1] Naimen, N., Frohlich, M. and Todesco, A. (1975) The Good Language Learner. TESL TALK, 6, 58-75.

[2] O’Malley, J. and Chamot, A. (1990) Learning Strategies in Second Language Acquisition. Cambridge University Press, Cambridge. http://dx.doi.org/10.1017/CBO9781139524490

[3] Cohen, D.A. (1998) Strategies in Learning and Using a Second Language. Longman Press, London.

[4] Gu, P.Y. and Johnson, R.K. (1996) Vocabulary Learning Strategies and Language Learning Outcomes. Language Learning, 46, 643-679. http://dx.doi.org/10.1111/j.1467-1770.1996.tb01355.x 